\title{
Atypical Presentations of Intracranial Hypotension: Comparison with Classic Spontaneous Intracranial Hypotension
}

\author{
(D)A.A. Capizzano, (D). Lai, (D). Kim, (D) Mizzo, Dl. Gray, (D) M.K. Smoot, and (D)T. Moritani
}

\begin{abstract}
BACKGROUND AND PURPOSE: Atypical clinical presentations of spontaneous intracranial hypotension include obtundation, memory deficits, dementia with frontotemporal features, parkinsonism, and ataxia The purpose of this study was to compare clinical and imaging features of spontaneous intracranial hypotension with typical-versus-atypical presentations.
\end{abstract}

MATERIALS AND METHODS: Clinical records and neuroimaging of patients with spontaneous intracranial hypotension from September 2005 to August 2014 were retrospectively evaluated. Patients with classic spontaneous intracranial hypotension ( $n=33$; mean age, $41.7 \pm$ 14.3 years) were compared with those with intracranial hypotension with atypical clinical presentation ( $n=8$; mean age, $55.9 \pm 14.1$ years) and 36 controls (mean age, $41.4 \pm 11.2$ years).

RESULTS: Patients with atypical spontaneous intracranial hypotension were older than those with classic spontaneous intracranial hypotension ( $55.9 \pm 14.1$ years versus $41.7 \pm 14.3$ years; $P=.018$ ). Symptom duration was shorter in classic compared with atypical spontaneous intracranial hypotension ( $3.78 \pm 7.18$ months versus $21.93 \pm 18.43$ months; $P=.015$ ). There was no significant difference in dural enhancement, subdural hematomas, or cerebellar tonsil herniation. Patients with atypical spontaneous intracranial hypotension had significantly more elongated anteroposterior midbrain diameter compared with those with classic spontaneous intracranial hypotension ( $33.6 \pm 2.9$ $\mathrm{mm}$ versus $27.3 \pm 2.9 \mathrm{~mm} ; P<.001)$ and shortened pontomammillary distance $(2.8 \pm 1 \mathrm{~mm}$ versus $5.15 \pm 1.5 \mathrm{~mm} ; P<.001)$. Patients with atypical spontaneous intracranial hypotension were less likely to become symptom-free, regardless of treatment, compared with those with classic spontaneous intracranial hypotension $\left(\chi^{2}=13.99, P<.001\right)$.

CONCLUSIONS: In this sample of 8 patients, atypical spontaneous intracranial hypotension was a more chronic syndrome compared with classic spontaneous intracranial hypotension, with more severe brain sagging, lower rates of clinical response, and frequent relapses. Awareness of atypical presentations of spontaneous intracranial hypotension is paramount.

ABBREVIATIONS: AP = average anteroposterior midbrain diameter; FBSS = frontotemporal brain sagging syndrome; $\mathrm{PM}=$ pontomammillary distance; $\mathrm{SIH}=$ spontaneous intracranial hypotension

S pontaneous intracranial hypotension (SIH) results from CSF hypovolemia, most commonly from a CSF spinal leak, and typically presents with orthostatic headache. ${ }^{1}$ On brain MR imaging, classic features of SIH are the following: downward brain

Received October 29, 2015; accepted after revision December 21.

From the Department of Radiology (A.A.C., T.M.), University of lowa Carver College of Medicine, lowa City, lowa; Department of Radiology (L.L.), Stanford University School of Medicine, Stanford, California; Department of Radiology (J.K.), University of Illinois at Chicago, Chicago, Illinois; Department of Neurological Sciences (M.R.), University of Nebraska Medical Center, Omaha, Nebraska; Department of Radiology (L.G.), Duke University Medical Center, Durham, North Carolina; and Department of Orthopaedic Surgery and Sports Medicine (K.S.), University of Kentucky, Lexington, Kentucky.

Please address correspondence to Aristides A. Capizzano, MD, Department of Radiology, Division of Neuroradiology, University of lowa Carver College of Medicine, 200 Hawkins Dr, IA 52242; e-mail: aristides-capizzano@uiowa.edu

三 Indicates article with supplemental on-line table.

http://dx.doi.org/10.3174/ajnr.A4706 sagging with effacement of perimesencephalic cisterns and pituitary and dural engorgement, which are sometimes associated with subdural hematoma. ${ }^{1,2}$ Recognition of SIH is very important for adequate management, which may include epidural blood patch when appropriate. Atypical clinical presentations of SIH have been sparsely reported in the literature, mostly in case report format, and include the following: obtundation, ${ }^{3}$ stupor, ${ }^{4}$ sensorineural deafness, ${ }^{5}$ short-term memory deficit, ${ }^{5}$ dementia with frontotemporal features, ${ }^{6-8}$ and parkinsonism and ataxia. ${ }^{9}$. An intriguing association of behavioral variant frontotemporal dementia-like symptoms with SIH has recently been reported as "frontotemporal brain sagging syndrome" (FBSS) on the basis of clinical-radiologic correlation. ${ }^{10}$

The prevalence of FBSS is thought to be very low, with the retrospective series from the Mayo Clinic consisting of 8 patients identified between January 1996 and July 2010. ${ }^{10}$ However, the 
true incidence of intracranial hypotension is most likely underestimated, particularly with atypical clinical presentations. Furthermore, an incorrect diagnosis of SIH is very common, such as misdiagnosis of SIH as Chiari I malformation, leading to improper patient management. ${ }^{11}$ Neuroradiologists play a key role in diagnosing classic and particularly atypical intracranial hypotension by recognizing the specific findings of brain sagging on MR imaging, detecting the level of CSF leak on spinal imaging, and guiding therapy accordingly. Therefore, the aim of this study was to illustrate different clinical presentations of intracranial hypotension that differ from the classic SIH syndrome and to assess their neuroimaging correlates and outcome. Specifically, the purpose of this study was to compare demographic, clinical, and brain imaging features of patients with classic SIH versus intracranial hypotension with atypical clinical presentations, including FBSS.

\section{MATERIALS AND METHODS}

An institutional review board-approved, retrospective review was performed of clinical records and brain MRI of patients evaluated for intracranial hypotension from September 2005 to August 2014 at the University of Iowa Carver College of Medicine. A waiver of consent was obtained from the local institutional review board. Only patients with brain MRI available for review were included. Patients were divided into 2 groups: classic spontaneous intracranial hypotension ( $\mathrm{SIH}, n=33$; mean age, $41.7 \pm 14.3$ years; 10 men, 23 women) and intracranial hypotension with atypical clinical presentation (atypical, $n=8$; mean age, $55.9 \pm$ 14.1 years; 5 men, 3 women). The control group consisted of 36 subjects with normal brain MRI findings, age- and sexmatched to patients with SIH (mean age, $41.4 \pm 11.2$ years; 12 men, 24 women). Patients with SIH presented with orthostatic headaches and brain MR imaging findings of brain sagging and dural enhancement with or without subdural fluid collections. Patients with atypical SIH were selected on the basis of clinical and imaging features of intracranial hypotension with atypical brain stem, cognitive, and/or behavioral symptoms previously referred to as FBSS, ${ }^{10}$ for which no other explanation for brain sagging could be obtained after extensive clinical, laboratory, and imaging assessment. The average clinical follow-up was $42.5 \pm 37.2$ months for atypical SIH and $18.2 \pm 23.7$ months for classic SIH.

All subjects were scanned with clinical 1.5T or 3T MR imaging scanners with clinical brain MR imaging protocols, including conventional pre- and postgadolinium T1-, T2-, and $\mathrm{T} 2{ }^{\star}-$ weighted sequences and an axial DTI sequence with 12 diffusion directions. The axial level of the midbrain was selected on 5-mmthick axial T2 images oriented parallel to the line connecting the inferiormost points of the genu and splenium of the corpus callosum at the level showing the red nuclei. The average anteroposterior midbrain diameter (AP) and pontomammillary distance (PM) were combined in the Sagging Index $=\mathrm{AP} / \mathrm{PM}$ (Fig 1), which was used as an imaging biomarker of the severity of brain sagging and not to diagnose SIH. The intraclass correlation coefficients for the 36 controls between 2 independent image readers were $0.86,0.81$, and 0.8 for the AP midbrain diameter, the PM distance, and the Sagging Index, respectively, indicating very good reproducibility. When available, spinal imaging (nuclear medicine cisternography, CT myelography, and/or whole-spine MR imaging) was assessed for determination of the site of CSF leak. Statistical analyses were performed with SPSS 21 (IBM, Armonk, New York). Nonparametric statistical analyses by using the Kruskal-Wallis or Mann-Whitney $U$ test as appropriate were performed. Distribution of discrete variables between groups was assessed with the $\chi^{2}$ test. A 2 -tailed $P$ value $<.05$ was used as a statistical significance threshold.

\section{RESULTS}

\section{Demographics and Clinical Data}

There were no significant differences in age or sex distribution between patients with classic SIH and controls (SIH: $41.7 \pm 14.3$ years, 70\% women versus controls: $41.4 \pm 11.2$ years, $67 \%$ women). Patients with atypical SIH were older than those with classic SIH (55.9 \pm 14.1 years versus $41.7 \pm 14.3$ years, $P=.018$ ). Among those with atypical SIH, there was a higher percentage of men (63\%) compared with classic SIH (30\%), but this trend in sex distribution did not reach statistical significance $\left(\chi^{2}=2.87, P=\right.$ $.09)$. No differences among the 3 groups were detected for body mass index $(P=.86)$. No neuropsychological assessment was performed for patients with classic SIH. A formal neurologic examination at the time of SIH symptoms was performed in 94\% (31/ 33) of patients with classic SIH. Classic SIH invariably presented with orthostatic headaches (ie, with worsening pain while standing up and improving on lying down), and 6 of these patients (18\%) had uni- or bilateral sixth nerve palsy, which resolved along with the headache. Symptom duration was $<1$ year for all classic SIH cases except for 1 woman with 10 years of positional headaches after delivery. When this latter patient was excluded, symptom duration was significantly shorter in classic SIH compared with atypical $\mathrm{SIH}(3.78 \pm 7.18$ months versus $21.93 \pm 18.43$ months, $P=.015)$.

The On-line Table summarizes the clinical features of 8 patients with atypical intracranial hypotension. Seven of 8 patients with atypical SIH had headache, which was mild in 2 of them. None of these patients had oculomotor abnormalities. Neuropsychological assessment of patients with atypical SIH yielded an atypical, not-otherwise-specified neuropsychological profile. Seven of 8 patients had anterograde memory impairment, which, in 4 cases, was associated with disinhibition, with 2 of these patients (cases 2 and 3 in the On-line Table) fulfilling the clinical diagnostic criteria for behavioral variant frontotemporal dementia. ${ }^{12}$ However, follow-up did not confirm this diagnosis: Neuropathologic assessment of patient 2 at postmortem examination did not show changes of frontotemporal dementia or any other neurodegenerative disease, while patient 3 showed clinical improvement after patching of spinal leaks, intraspinal fluid infusions, and ultimately a spinal operation. Notably, 7 of 8 patients with atypical SIH had daytime hypersomnia. Five of 8 had gait anomalies with ataxic gait and imbalance resulting in frequent falls. Four of 8 had movement disorders: Two had facial grimacing, 1 had upper limb chorea, and 1 had resting tremor. Finally, 3 of 8 patients with atypical SIH had dysarthria and dysphagia, and 2 of 8 had stereotyped vocal tics. 


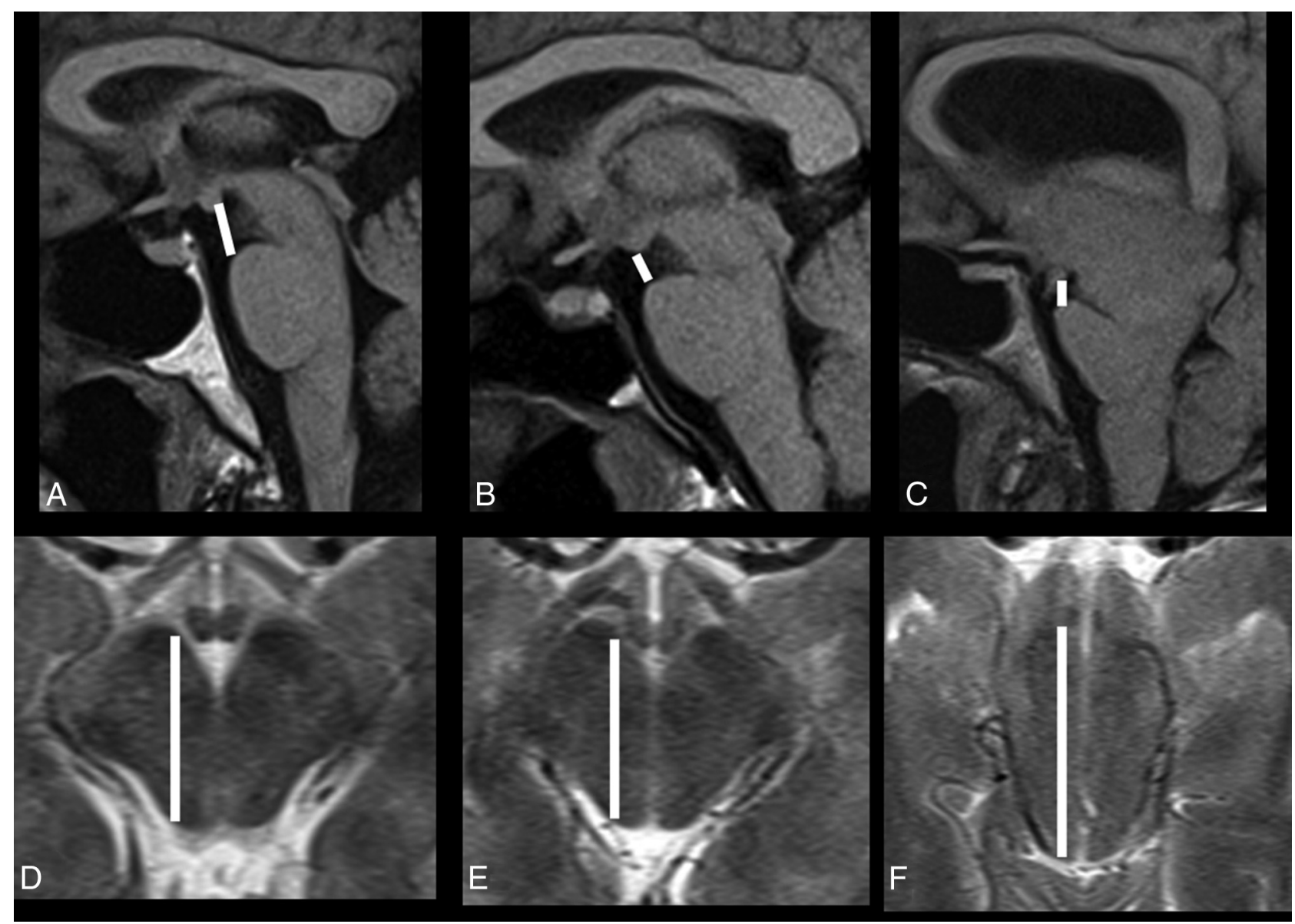

FIG 1. Pontomammillary distance on sagittal $T 1(A-C)$ and anteroposterior midbrain diameter on axial T2-weighted MRI $(D-F)$ in a healthy control $(A$ and $D)$, and patients with classic $\mathrm{SIH}(B$ and $E)$ and atypical SIH (C and $F)$.

\section{Imaging Findings: Classic SIH versus Atypical SIH}

Twenty-seven $(82 \%)$ patients with classic SIH had dural enhancement, and $14(42 \%)$ had subdural fluid collections. Patients with atypical SIH had a lower prevalence of dural enhancement (62\%) and subdural hematomas (25\%) compared with those with classic $\mathrm{SIH}$, but this was not statistically significant. Furthermore, no significant difference in the extent of herniation of the cerebellar tonsils below the plane of the foramen magnum was detected (SIH: $2.25 \pm 2.96 \mathrm{~mm}$ versus atypical: $5.96 \pm 6.44 \mathrm{~mm} ; P=.2$ ). Patients with atypical SIH had more distorted midbrain anatomy at the level of the tentorial incisura compared with those with classic SIH, with significantly elongated anteroposterior midbrain diameter (atypical: $33.6 \pm 2.9 \mathrm{~mm}$ versus SIH: $27.3 \pm 2.9 \mathrm{~mm}$, $P<.001$ ) and shortened pontomammillary distance (atypical: $2.8 \pm 1 \mathrm{~mm}$ versus SIH: $5.15 \pm 1.5 \mathrm{~mm} ; P<.001)$. The combination of the latter 2 measurements in the Sagging Index (AP/PM) showed highly significant differences between the 2 groups (atypical: $14.98 \pm 8.6$ versus SIH: $6.02 \pm 2.25, P<.001$ ) (Fig 2). When we used a cutoff value of 10 in the Sagging Index, only 3 patients were misclassified, with a correct classification of 93\%. Finally, 4 of $8(50 \%)$ patients with atypical SIH had CSF leaks on spinal imaging (spine MR imaging, CT myelography, and/or radioisotope cisternography), while 19 of 33 (57\%) patients with classic SIH had CSF leaks on spinal imaging, with no significant statistical difference between the groups.

\section{Treatment and Follow-Up}

All 30 patients with classic SIH with follow-up clinical data available found their symptoms resolved following blood patch (13/ $30)$, burr-hole for subdural drainage (2/30), spinal dural surgical repair (1/30), or medical treatment alone (14/30). There was a significantly lower chance of symptom resolution for patients with atypical compared with classic $\operatorname{SIH}\left(\chi^{2}=13.99, P<.001\right)$. Four of 7 patients with atypical SIH with available follow-up showed continued clinical improvement; these were treated with epidural blood patch (case 6 in the On-line Table), multiple epidural blood and glue patches and CSF infusions followed by surgery (case 3), thoracic dural cyst ligation (case 4), and burr-hole drainage of a subdural hematoma (case 8). The remaining 3 patients with atypical SIH did not show clinical improvement: One was treated with Chiari decompression and had stable symptoms of ataxia and resting tremor (case 5), and 2 patients died from unrelated conditions (cases 2 and 7). One patient with atypical $\mathrm{SIH}$ was lost on follow-up due to relocation before receiving specific treatment (case 1). Three patients with atypical SIH died of unrelated conditions (case 2, from myocardial infarction; case 4 died at another institution; and case 7 , from complications of trauma and anticoagulation). Postmortem examination with neuropathologic assessment of the brain for neurodegenerative disease was performed in case 2 and did not show evidence of frontotemporal dementia pathology or any other neurodegenerative disease. 


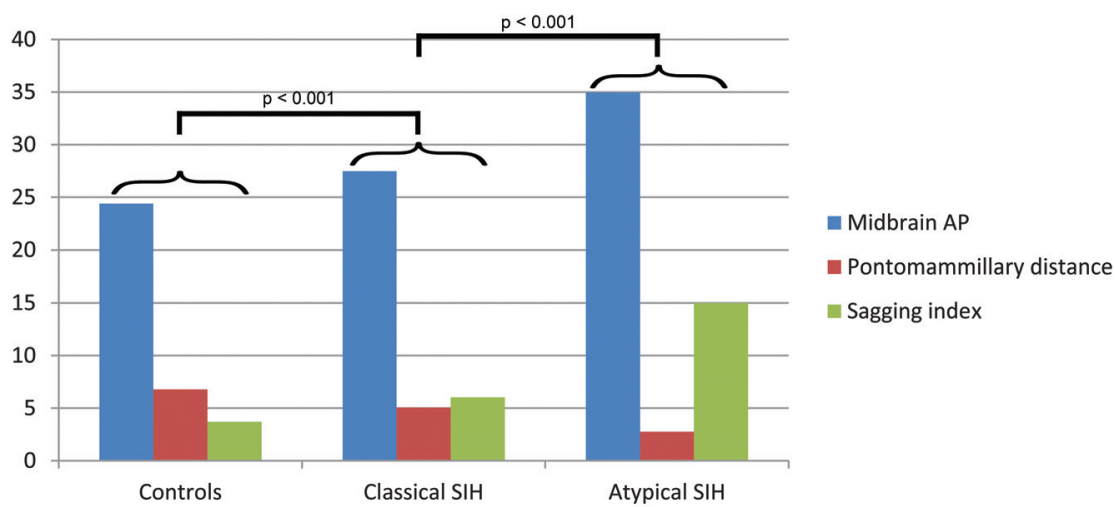

FIG 2. Pontomammillary distance, anteroposterior midbrain diameter, and Sagging Index in patients with atypical and classic SIH and controls.

\section{DISCUSSION}

Patients with atypical SIH were older than those with classic SIH and had a trend toward higher representation of men, while women are more commonly affected than men in classic SIH. ${ }^{2}$ Patients with atypical SIH had a longer clinical history and lower odds of becoming symptom-free compared with those with classic SIH. The degree of brain sagging at the tentorial incisura was more severe in patients with atypical-versus-classic $\mathrm{SIH}$ without significant differences in other radiographic signs of intracranial hypotension, such as dural enhancement, fluid collections, or cerebellar tonsillar herniation. A Sagging Index higher than 10 should prompt consideration of intracranial hypotension in subjects with atypical clinical presentations.

Case 3 (On-line Table) exemplifies the challenges associated with atypical SIH. A 57-year-old man presented with behavioral changes, anterograde amnesia, headache, hypersomnia, dysphagia, ataxia, dysarthria, and gait instability and was diagnosed with behavioral variant frontotemporal dementia. He had a history of mitral valve prolapse, suggesting connective tissue laxity. Brain MR imaging showed severe brain sagging but no cortical atrophy. On CT myelography, 2 small CSF leaks at T10-T11 and T11-T12 were seen and treated with fluoroscopically guided autologous blood patches (3 times), followed by transient clinical improvement. Neuropsychological evaluation showed mild deficits in processing speed, attention, and concentration, with improvement after blood patches. His symptoms relapsed. Therefore, he was referred to another institution, where on dynamic CT myelogram, multiple CSF leaks at the cervicothoracic neuroforamina were detected and patched under CT guidance first with blood and later with glue; and he also received artificial CSF infusions. The patient's gait and speech improved after the spinal procedures, but symptoms eventually relapsed.

Three years after presentation, he underwent endoscopic third ventriculostomy. Although intracranial hypotension is not an indication for endoscopic third ventriculostomy, the rationale was the presence of a gradient between low intracranial pressure (as measured in an outside ventriculostomy) and normal spinal pressure measured at spinal procedures. Because there was no evidence of a blockade at the foramen magnum, the neurosurgeons hypothesized that the endoscopic third ventriculostomy would equalize pressures between the ventricular system and the subarachnoid spaces of the basal cisterns and spine. His gait and speech improved for 2 weeks, but full relapse followed. Brain MR imaging showed stable sagging and undetectable CSF flow in the aqueduct (Fig $3 A-C$ ). Technetium Tc99m SPECT spinal CSF leak study findings were negative. Four months after ventriculostomy, he underwent T9 through T12 laminoplasties, levels where small CSF leaks had been seen on myelography, but these were not found at the operation. Exposure of the arachnoid revealed adhesions, which were partially released, and arachnoid biopsy reported fibrosis without inflammation or granulomas. Fibrin sealant (TISSEEL; Baxter Healthcare, Deerfield, Illinois) was applied to seal the dura. Six months after the operation, he had continued clinical improvement with normalization of gait and speech, recovery of the gag reflex, and behavioral and cognitive recovery to baseline. Brain MR imaging showed resolution of brain sagging and recovery of aqueductal CSF flow for the first time at the 4-year follow-up (Fig 3D-F).

The etiology of SIH has been linked to weakness of the spinal dura, resulting in spinal CSF leak, in some cases associated with hereditary connective tissue disorders. ${ }^{13} \mathrm{CSF}$ venous fistulas have also recently been implicated in a few cases. ${ }^{14}$ Management includes medical treatment (rest, hydration, caffeine) with escalation to spinal epidural patching when symptoms persist. The success rate of the epidural blood patch for SIH, however, is about $30 \%$, far lower than that for post-lumbar puncture headache, ${ }^{15}$ and patients with SIH may require repeat spinal procedures. Surgical repair of the spinal dural defect by using different techniques ${ }^{16}$ is reserved for cases that fail to respond to conservative measures. As in case 3, a CSF leak was not seen on surgery in 6 of 10 patients with SIH in whom epidural patching with muscle and Gelfoam (Pfizer, New York, New York) was, nevertheless, 100\% successful in treating headache. ${ }^{16}$ Neuroradiologists make key contributions in the diagnosis and management of classic and particularly atypical SIH. The latter has a longer clinical history probably because the atypical clinical features distract clinicians from the consideration of intracranial hypotension. Furthermore, clinical response is less favorable in patients with atypical compared with those with classic SIH. This could result from structural weakness of the dura or insufficient physiologic adaptation to chronic CSF hypovolemia. ${ }^{1}$

Atypical clinical presentations of SIH can be grouped into 4 domains: cognitive-behavioral, lower cranial nerve, imbalancegait, and arousal deficits. Typically patients present with a combination of these symptom complexes. Neuropsychologically, patients with atypical SIH present with a not-otherwise-specified profile suggestive of a subcortical type of cognitive impairment ${ }^{17}$ with slowed information processing, preserved language, and anterograde amnesia. FBSS is a rare, recently recognized syndrome in which behavioral variant frontotemporal dementia-like symptoms present in the context of intracranial hypotension, though an unidentified etiology can not be ruled out, ${ }^{10}$ and therefore should be included among the causes of potentially reversible cog- 


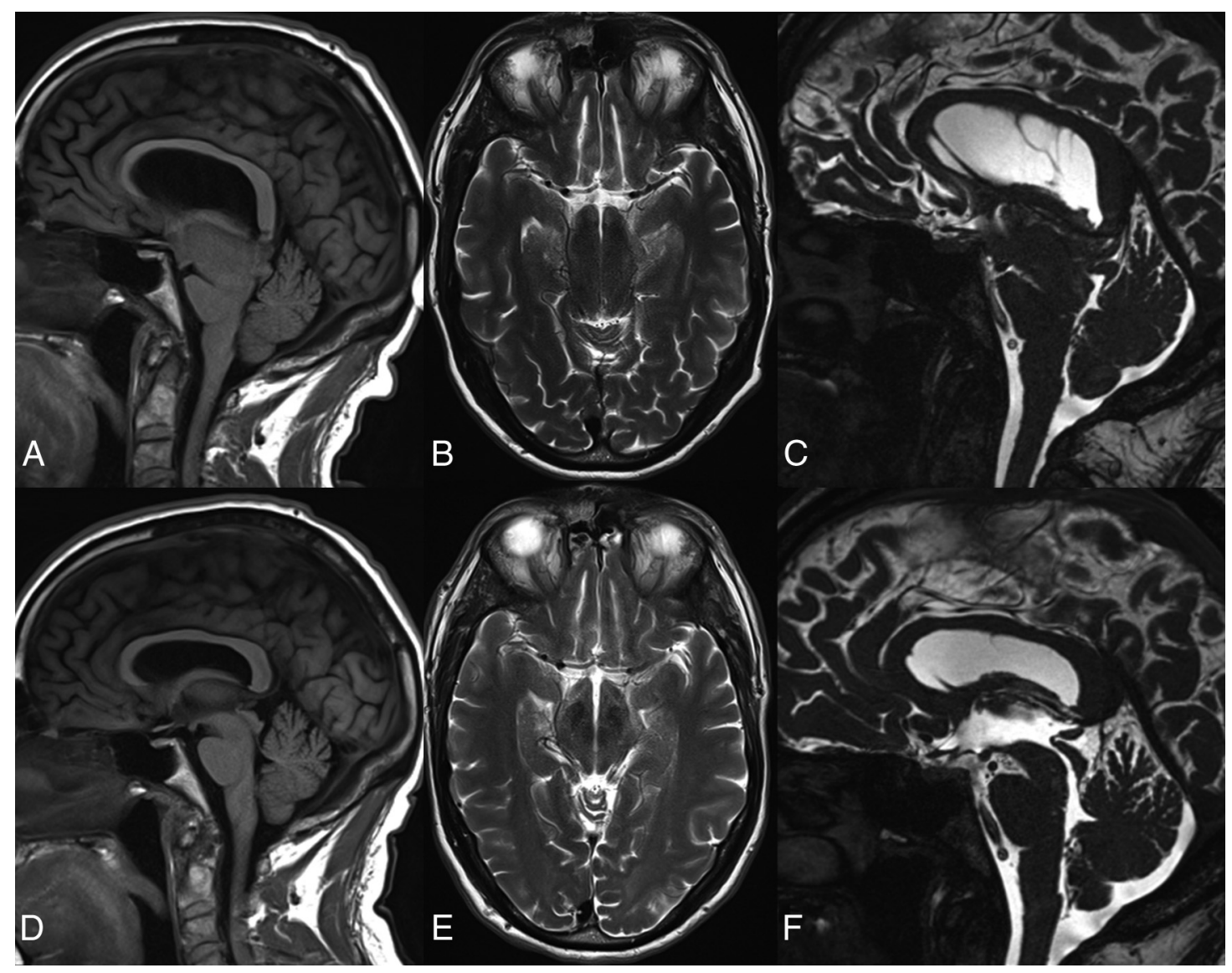

FIG 3. Preoperative $(A-C)$ and postoperative $(D-F)$ sagittal T1 ( $A$ and $D)$, axial T2 ( $B$ and $E)$, and sagittal CISS (C and $F)$ brain MRI in case 3 . Note postoperative resolution of brain sagging and recovery of aqueductal CSF lumen.

nitive impairment. Age of onset and male predominance in FBSS coincide with those in behavioral variant frontotemporal dementia. On imaging, FBSS lacks the typical cortical atrophy seen in frontotemporal dementia, presenting with brain sagging instead. ${ }^{10}$ In the few reported cases of neuropathologic examination, ${ }^{10}$ including our case 2 , no specific neurodegenerative disease was found. We propose that FBSS, with its predominant neurocognitive presentation, should be included in the more inclusive category of atypical SIH.

Apart from cognitive and behavioral deficits, patients with atypical SIH frequently present with gait disturbance, imbalance, dysarthria, dysphagia, daytime somnolence, and movement disorders, which are not typical features of behavioral variant frontotemporal dementia. These clinical manifestations suggest midbrain and/or brain stem involvement, corresponding to the level of brain sagging and anatomic distortion. The frequent association of daytime somnolence in our sample of patients with atypical SIH and in the published series of FBSS $^{10}$ points to involvement of the brain stem reticular activation system. In agreement with this notion, reversibility of obtundation from SIH has been highly time-correlated to recovery of a normal intracranial pressure waveform with reactive pupils after intrathecal saline infusion, supporting a mesencephalic level. ${ }^{3}$ Ataxia and dysarthria likely result from stretching of the superior cerebellar peduncles. Some of the symptoms associated with atypical SIH respond promptly to intrathecal saline infusion, such as the transient gait improvements noted in our case 3. Half of our patients with atypical SIH presented with involuntary movement disorders including a case of upper limb chorea with negative neuropathologic findings for Huntington disease or other neurodegenerative diseases. Parkinsonism and ataxia with recovery after blood patch were reported in a case of SIH. ${ }^{9}$ Finally, oculomotor abnormalities were also reported in FBSS, ${ }^{10}$ though these were not observed in our sample.

The cardinal radiologic feature of $\mathrm{SIH}$ is brain sagging at the tentorial incisura, resulting in the following morphologic distortions: approximation of both thalami, caudal displacement of the mammillary bodies with shortened pontomammillary distance, and anteroposterior elongation from lateral compression of the midbrain at the tentorial incisura. The Monro-Kellie doctrine ${ }^{18}$ explains the imaging findings of pachymeningeal enhancement (from venous engorgement) and subdural fluid collections as compensatory mechanisms to preserve the homeostasis of intracranial volume in the setting of a CSF volume deficit. ${ }^{19}$ Atypical $\mathrm{SIH}$ is a more chronic and severe brain sagging syndrome compared with classic $\mathrm{SIH}$, as evidenced by the significantly higher Sagging Index (Fig 2) in the absence of other neuroradiologic differences. Compensatory mechanisms to CSF hypovolemia might become insufficient in such chronic conditions to prevent the observed parenchymal stretching across the tentorial incisura. On the basis of imaging and clinical features, we propose that the anatomic site of involvement in atypical SIH corresponds to the 
structures adjacent to the tentorial incisura: the medial thalamus, hypothalamus, midbrain tegmentum, and upper brain stem.

The main limitation of this study is the low number of patients with atypical SIH, inherent in the rarity of this syndrome and/or its underdiagnosis. Another limitation is the incomplete clinical and imaging follow-up related to the retrospective nature of this study. One patient with atypical SIH was treated with Chiari decompression based on neurosurgical indications, with no clinical improvement. Finally, no systematic information was available on connective tissue anomalies, which could underlie the chronicity and frequent relapses of patients with atypical SIH.

\section{CONCLUSIONS}

Atypical SIH, including FBSS, is a complex syndrome that should be included in the category of potentially reversible, treatable causes of dementia. It is therefore paramount to suspect it clinically and to confirm it on brain MR imaging, for which a Sagging Index of $\geq 10$ is highly suggestive. Older age and male sex are more common in atypical compared with classic SIH. The longer clinical duration and less favorable outcome of atypical SIH may relate to a weaker dura from an underlying connective tissue anomaly and/or insufficient physiologic adaptation to CSF hypovolemia, leading to parenchymal stretching with brain sagging. The potential for reversibility of symptoms and signs, even in chronic cases with atypical presentation after tamponade of the CSF leak, underscores the relevant role of neuroradiologists in diagnosing and managing $\mathrm{SIH}$.

\section{REFERENCES}

1. Schievink WI, Deline CR. Headache secondary to intracranial hypotension. Curr Pain Headache Rep 2014;18:457 CrossRef Medline

2. Urbach $H$. Intracranial hypotension: clinical presentation, imaging findings, and imaging-guided therapy. Curr Opin Neurol 2014;27: 414-24 CrossRef Medline

3. Binder DK, Dillon WP, Fishman RA, et al. Intrathecal saline infusion in the treatment of obtundation associated with spontaneous intracranial hypotension: technical case report. Neurosurgery 2002;51: 830-36; discussion 836-37 Medline

4. Pleasure SJ, Abosch A, Friedman J, et al. Spontaneous intracranial hypotension resulting in stupor caused by diencephalic compression. Neurology 1998;50:1854-57 CrossRef Medline

5. Peng PW. Intracranial hypotension with severe neurological symptoms resolved by epidural blood patch. Can J Neurol Sci 2004;31: 569-71 CrossRef Medline

6. Sayao AL, Heran MK, Chapman K, et al. Intracranial hypotension causing reversible frontotemporal dementia and coma. $\mathrm{Can} \mathrm{J} \mathrm{Neu-}$ rol Sci 2009;36:252-56 CrossRef Medline

7. Hong M, Shah GV, Adams KM, et al. Spontaneous intracranial hypotension causing reversible frontotemporal dementia. Neurology 2002;58:1285-87 CrossRef Medline

8. Walker L, DeMeulemeester C. Spontaneous intracranial hypotension masquerading as frontotemporal dementia. Clin Neuropsychol 2008;22:1035-53 CrossRef Medline

9. Pakiam AS, Lee C, Lang AE. Intracranial hypotension with parkinsonism, ataxia, and bulbar weakness. Arch Neurol 1999;56:869-72 CrossRef Medline

10. Wicklund MR, Mokri B, Drubach DA, et al. Frontotemporal brain sagging syndrome: an SIH-like presentation mimicking FTD. Neurology 2011;76:1377-82 CrossRef Medline

11. Schievink WI. Misdiagnosis of spontaneous intracranial hypotension. Arch Neurol 2003;60:1713-18 CrossRef Medline

12. Neary D, Snowden JS, Gustafson L, et al. Frontotemporal lobar degeneration: a consensus on clinical diagnostic criteria. Neurology 1998;51:1546-54 CrossRef Medline

13. Schievink WI, Gordon OK, Tourje J. Connective tissue disorders with spontaneous spinal cerebrospinal fluid leaks and intracranial hypotension: a prospective study. Neurosurgery 2004;54:65-70; discussion 70-71 CrossRef Medline

14. Schievink WI, Moser FG, Maya MM. CSF-venous fistula in spontaneous intracranial hypotension. Neurology 2014;83:472-73 CrossRef Medline

15. Mokri B. Spontaneous CSF leaks: low CSF volume syndromes. Neurol Clin 2014;32:397-422 CrossRef Medline

16. Schievink WI, Morreale VM, Atkinson JL, et al. Surgical treatment of spontaneous spinal cerebrospinal fluid leaks. J Neurosurg 1998;88: 243-46 CrossRef Medline

17. Cummings JL. Subcortical dementia: neuropsychology, neuropsychiatry, and pathophysiology. Br J Psychiatry 1986;149:682-97 Medline

18. Mokri B. The Monro-Kellie hypothesis: applications in CSF volume depletion. Neurology 2001;56:1746-48 CrossRef Medline

19. Fishman RA, Dillon WP. Dural enhancement and cerebral displacement secondary to intracranial hypotension. Neurology 1993;43: 609-11 CrossRef Medline 